\title{
L-Carnitine improves gastrointestinal disorders and altered the intestinal microbiota in hemodialysis patients
}

\author{
Junichiro IRIE1, Yoshihiko KANNO2*, Rieko KIKUCHI', Tadashi YOSHIDA, Seizo MURAI", \\ Miwako WATANABE ${ }^{5}$, Hiroshi ITOH $^{1}$ and Matsuhiko HAYASHI ${ }^{3}$ \\ ${ }^{1}$ Division of Endocrinology, Metabolism and Nephrology, Department of Internal Medicine, School of Medicine, Keio University, \\ Shinjuku-ku, Tokyo 160-8582, Japan \\ ${ }^{2}$ Department of Nephrology, Tokyo Medical University, 6-7-1 Nishishinjuku, Shinjuku, Tokyo 160-0023, Japan \\ ${ }^{3}$ Apheresis and Dialysis Center, School of Medicine, Keio University, Tokyo 160-8582, Japan \\ 4 lidabashi Murai Clinic, Tokyo 162-0822, Japan \\ ${ }^{5}$ Tokyo Midtown Medical Center, Tokyo 107-6206, Japan
}

Received April 12, 2016; Accepted October 13, 2016; Published online in J-STAGE November 3, 2016

Patients receiving hemodialysis also manifest gastrointestinal symptoms, such as constipation, caused by restriction of water intake and the loss of body water balance. Because dietary carnitine deficiency is considered to cause smooth muscle dysmotility of the gastrointestinal tract similarly to that in skeletal muscles, carnitine deficiency in hemodialysis patients may be one cause of gastrointestinal discomfort and dysfunctions. We performed a multicenter nonrandomized single-arm prospective clinical trial. Fifteen Japanese patients receiving hemodialysis were administered L-carnitine tablets $(900 \mathrm{mg})$ for 3 months, and clinical and biochemical analyses were performed before and after treatment. The serum total carnitine level was increased significantly by supplementation with L-carnitine for 3 months (from $40.9 \pm$ $2.6 \mu \mathrm{mol} / \mathrm{l}$ to $172.3 \pm \mathbf{1 9 . 0} \mu \mathrm{mol} / \mathrm{l}, \mathrm{p}<\mathbf{0 . 0 5}$ ). The myasthenia score was decreased significantly by the supplementation (from $1.3 \pm 0.3$ to $0.8 \pm \mathbf{0 . 2}, \mathrm{p}<\mathbf{0 . 0 5}$ ). The frequency of passing stool tended to increase with the treatment for 3 months (from $4.2 \pm 0.5$ times/week to $4.8 \pm 0.5$ times/week). A phyla-level analysis of the microbiota showed that the composition of the individual microbiota was not different between before and after supplementation. A genus-level analysis, however, revealed that the relative abundance of genus Clostridium subcluster 4 was significantly decreased by the supplementation (from $7.7 \pm 1.9 \%$ to $4.7 \pm 1.3 \%$, p $<0.05$ ). Oral supplementation of L-carnitine to the patients receiving hemodialysis improved not only their muscle discomfort but also their gastrointestinal disorders and microbiota, although its effect on the prognosis of hemodialysis patients should be further investigated.

Key words: carnitine, Clostridium, constipation, microbiota

\section{INTRODUCTION}

Patients receiving hemodialysis tend to lack essential nutrients because of the dietary restriction and loss caused by dialysis therapy, resulting in the development of various clinical symptoms owing to the deficiencies. Carnitine is an essential nutrient that transports fatty acid into mitochondria and is stored mainly in muscles. Recent findings have revealed that patients receiving hemodialysis have a lower serum carnitine level and

\footnotetext{
*Corresponding author. Yoshihiko Kanno, Department of Nephrology, Tokyo Medical University, 6-7-1 Nishishinjuku, Shinjuku, Tokyo 160-0023, Japan. Phone: +81-3-3342-6111; Fax: +81-33342-2650, E-mail: kannoyh@tokyo-med.ac.jp

(C)2017 BMFH Press

This is an open-access article distributed under the terms of the Creative Commons Attribution Non-Commercial No Derivatives (bync-nd) License $<$ http://creativecommons.org/licenses/by-nc-nd/4.0/>.
}

lower carnitine pool in their muscles, indicating carnitine deficiency, and that L-carnitine supplementation improves their skeletal muscle discomfort and fatigue [1].

Patients receiving hemodialysis also manifest gastrointestinal symptoms such as constipation [2]. The restriction of water intake and the loss of body water balance may induce constipation, and it might elevate blood urea nitrogen (BUN) level in hemodialysis patients. Because dietary carnitine deficiency in infancy is considered to cause smooth muscle dysmotility of the gastrointestinal tract similarly to that in their skeletal muscles in the extremities, carnitine deficiency in hemodialysis patients may be one cause of gastrointestinal discomfort and dysfunctions [3]. However, the effects of carnitine supplementation on hemodialysis patients with gastrointestinal disorders remain to be evaluated [4]. Recently, it has been sensationally reported that CKD 
and/or uremia alters the human gut microbiota $[5,6]$.

Therefore, we investigated the changes in the clinical symptoms, metabolic parameters, and intestinal microbiota of chronic hemodialysis patients who received L-carnitine supplementation.

\section{SUBJECTS AND METHODS}

We performed a multicenter nonrandomized singlearm prospective clinical trial of outpatients receiving hemodialysis at Keio University Hospital and Murai Clinic, Japan. We enrolled 15 patients who had been receiving hemodialysis, and written informed consents was obtained from all the patients. L-carnitine tablets (900 mg) were administered for 3 months, and clinical and biochemical analyses were performed before and after the treatment. The conditions of hemodialysis (mode, dialysis hours per week, membrane surface area and material, and blood flow) in each patient were not changed throughout the study period. This study was conducted in accordance with the Declaration of Helsinki and was approved by the Ethics Review Board of Keio University (No. 2011-366, March 26, 2012). This study was also registered as a clinical study in the University Hospital Medical Information Network Clinical Trials Registry (UMIN-CTR: No. UMIN000007677).

Body weight increase and pre- and postdialysis session blood pressures were calculated as the mean of 6 dialysis sessions. The frequency of passing stool per a week was counted before and after L-carnitine treatment. Myasthenia was evaluated using the following scale: 1 , sometimes happen after dialysis and disappears within $30 \mathrm{~min}$; 2, always happens after dialysis and continue for 2 hours; 3 , always happens during dialysis and continue for 4 hours or longer. Blood samples were drawn from the arterial site of the arteriovenous fistula at the start or end of each dialysis session after a 2-day interval at 0 and 3 months after enrolment. Blood cell counts and the levels of BUN, serum creatinine, total protein, albumin, hemoglobin, and brain natriuretic peptide were measured by standard laboratory techniques using an autoanalyzer. Total, free, and acyl carnitine measurements were entrusted to Bio-Medical Laboratories, Inc. (Tokyo, Japan), and measured by enzyme cycling methods. Insulin resistance was assessed using plasma immunoreactive insulin (IRI) and homeostasis model assessment for insulin resistance (HOMA-R), with HOMA-R = fasting glucose $(\mathrm{mg} / \mathrm{dl}) \times$ fasting IRI $(\mathrm{mU} / \mathrm{ml}) / 405$ [7] .

Extraction and analysis of fecal microbiota were performed as reported previously [8]. Briefly, fecal samples were suspended in sterile distilled water by vigorous shaking, and the supernatant was transferred to a $0.5 \mathrm{ml}$ tube containing glass beads $(0.1 \mathrm{~mm}$ diameter), before being spun at 5,000 rpm for $3 \mathrm{~min}$ in a mini-bead beater (BioSpec Products, Bartlesville, OK, USA). DNA was extracted from the bead-treated suspension using phenol-chloroform extraction and isopropanol precipitation.

To enable terminal restriction fragment length polymorphism (T-RFLP) analysis, PCR was performed using the total fecal DNA and $25 \mu \mathrm{M}$ primers for labelled 516f (TGCCAGCAGCCGCGGTA) and 1510r (GGTTACCTTGTTACGACTT). The PCR conditions were as follows: $95^{\circ} \mathrm{C}$ for $15 \mathrm{~min} ; 30$ cycles of $95^{\circ} \mathrm{C}$ for $30 \mathrm{sec}, 50^{\circ} \mathrm{C}$ for $30 \mathrm{sec}$, and $72^{\circ} \mathrm{C}$ for $2 \mathrm{~min}$; and a final extension step of $72^{\circ} \mathrm{C}$ for $10 \mathrm{~min}$. The resultant amplicons were digested with $2 \mathrm{U}$ BslI (New England Biolabs Japan, Tokyo, Japan) for $1 \mathrm{~h}$ and then fractionated using an automated sequence analyzer (ABI PRISM 3130xl Genetic Analyzer, Applied Biosystems, Foster City, CA, USA). The lengths and peak areas of the fragments were determined using GeneMapper software (Applied Biosystems, Foster City, CA, USA). The relative abundance of operational taxonomic units (OTUs) was calculated by dividing the peak area of each OTU by the sum of all the peak areas. This T-RFLP analysis revealed the relative abundances of the phyla of Firmicutes, Bacteroidetes, Actinobacteria, and Proteobacteria, the order of Lactobacillales, and the genus of Clostridium subcluster 4 .

The relative abundances of Clostridium subcluster 4 of bacteria in the fecal samples were evaluated using quantitative PCR, which was performed using SYBR Green (Takara Bio, Kusatsu, Shiga, Japan) and an Mx3000P real-time PCR system (Agilent Technologies Japan Ltd., Tokyo, Japan), as reported previously [9]. The amplification procedure consisted of 1 cycle of $95^{\circ} \mathrm{C}$ for $10 \mathrm{~min}$, followed by 40 cycles of $95^{\circ} \mathrm{C}$ for $15 \mathrm{sec}$ and $60^{\circ} \mathrm{C}$ for $1 \mathrm{~min}$. All experiments were performed in duplicate, and melting curve analysis was performed after the amplification stage to exclude nontarget reactions. The frequencies of specific bacteria were calculated as ratios of the total number of bacteria. The total number of bacteria was assessed using universal primers for total bacteria $[10,11]$.

Data were expressed as mean \pm standard error values. The significance of differences between 2 groups was examined using Student t-test or the Wilcoxon signedrank test. P-values of $<0.05$ were considered to indicate a statistically significant difference. 
Table 1. Clinical profile of the patients before and after oral L-carnitine supplementation

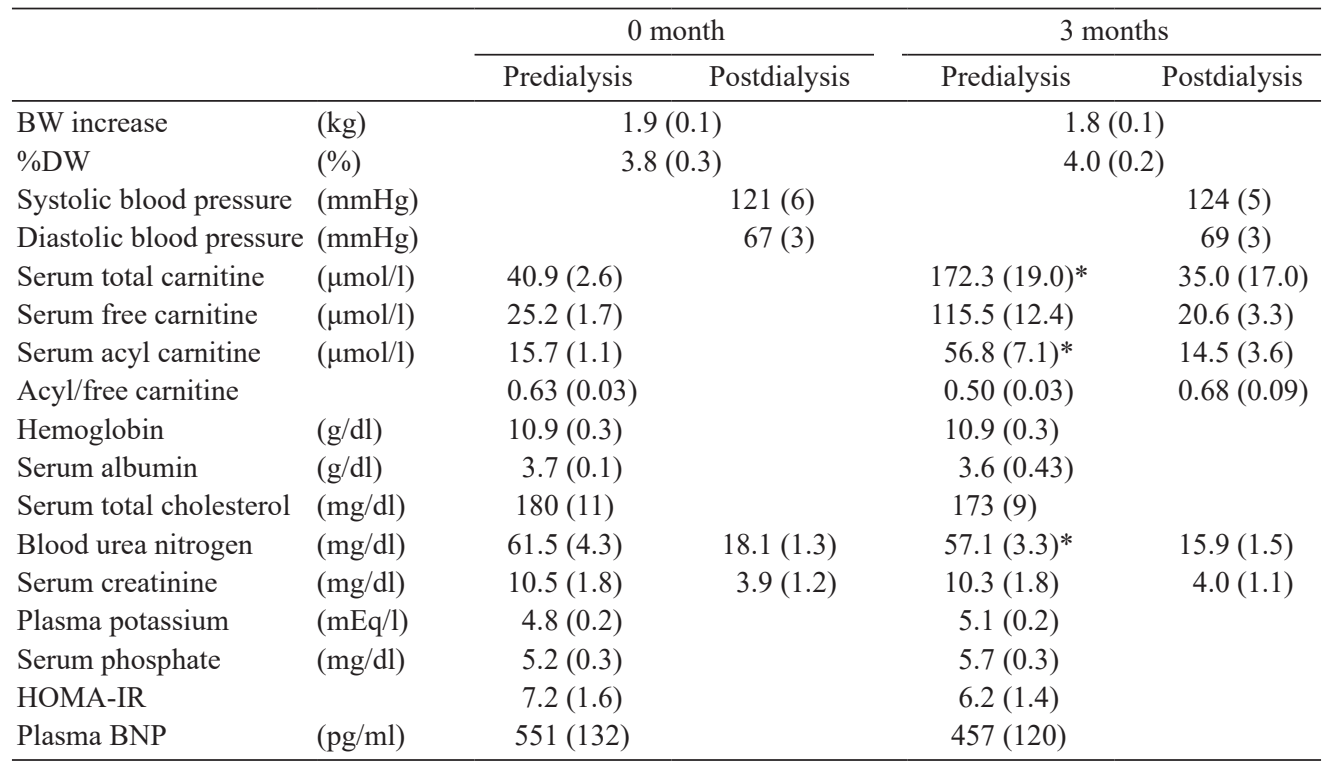

Data are expressed as means (SEM). The comparisons between the carnitine values were carried out using the predialysis values. ${ }^{*} \mathrm{p}<0.05$ compared with 0 month.

BW: body weight; DW: dry weight for dialysis session; HOMA-IR: homeostasis model assessment for insulin resistance; BNP: brain natriuretic peptide.

\section{RESULTS}

Fifteen Japanese patients receiving hemodialysis completed the study. They consisted of 6 men and 9 women aged $72 \pm 10$ years. They had a dialysis history of $12 \pm 7.9$ years, and 4 of the patients had diabetes mellitus. The patients' characteristics at baseline and after 3 months of treatment are shown in Table 1. Seven patients used laxative agents at study registration. The serum total carnitine level was increased significantly by supplementation with carnitine for 3 months (from $40.9 \pm 2.6 \mu \mathrm{mol} / 1$ to $172.3 \pm 19.0 \mu \mathrm{mol} / 1, \mathrm{p}<0.05$ ). This finding shows that the patients adhered to the treatment of carnitine supplementation. Consistent with previous reports, the myasthenia score was decreased significantly by the administration of L-carnitine (from $1.3 \pm 0.3$ to $0.8 \pm 0.2, \mathrm{p}<0.05)$. These findings indicated that the oral L-carnitine supplementation effectively remedied the deficiency of the systemic carnitine pool and the clinical symptoms caused by the deficiency in our patients.

Next, the effects of carnitine supplementation on the gastrointestinal tract were investigated. The frequency of passing stool per week was examined before and after supplementation. The frequency of passing stool tended to increase with treatment for 3 months (from $4.2 \pm 0.5$ times/week to $4.8 \pm 0.5$ times/week; not significantly different). Seven of the 15 subjects took laxatives before the administration of carnitine, whereas 5 of the 15 subjects took laxatives after the administration of carnitine. Regarding the resolution of constipation, the predialysis BUN level decreased significantly after 3 months of supplementation (from $61.5 \pm 4.3 \mathrm{mg} / \mathrm{dl}$ to $57.1 \pm 3.3 \mathrm{mg} / \mathrm{dl}, \mathrm{p}<0.05)$. Dry weight and the increase in body weight between the patients' hemodialysis sessions (from $1.9 \pm 0.1 \mathrm{~kg}$ to $1.8 \pm 0.1 \mathrm{~kg}$ ) were not significantly changed after supplementation, indicating that their nutritional status did not markedly change for 3 months. Serum creatinine levels were not significantly changed during the study, indicating that the effects of hemodialysis were also not changed. Therefore, the decrease in the BUN level after supplementation might have been attributable to the changes in their gastrointestinal conditions.

Moreover, because the intestinal microbiota can be modulated by intestinal conditions, analyses of the intestinal microbiota were conducted. A phyla-level analysis of the microbiota showed that the composition of the individual microbiota was not different between before and after supplementation (Fig. 1). This finding was consistent with a previous report indicating that the composition of the intestinal microbiota is preserved individually [12]. An order- and genus-level analysis, however, revealed a significant decrease in the relative abundances of the genus Clostridium subcluster 4 

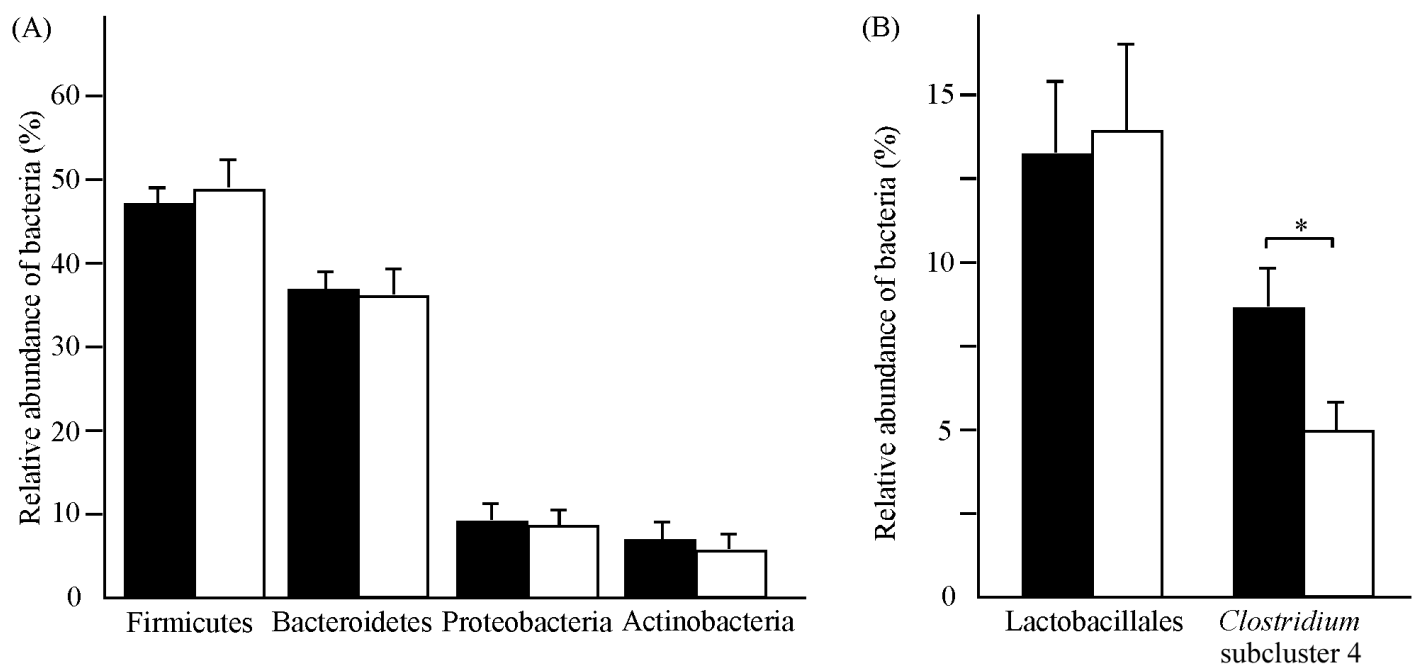

Fig. 1. The relative abundance of intestinal bacteria in the hemodialysis patients before and after oral L-carnitine supplementation for 3 months.

Fifteen patients who were receiving hemodialysis and had low serum carnitine levels were administered L-carnitine $(900 \mathrm{mg}$ ) for 3 months, and stool samples were collected before and after treatment. Bacterial 16S rDNA was extracted from the fecal samples, amplified by PCR, and subjected to terminal restriction fragment length polymorphism (T-RFLP) analysis. (A) The relative abundances of each speculated bacterial phyla to total bacteria are shown as means \pm SEM. (B) The relative abundances of Lactobacillales and Clostridium subcluster 4 are shown as means \pm SEM. Black bar, before treatment; white bar, after treatment.

caused by the supplementation treatment (from $7.7 \pm$ $1.9 \%$ to $4.7 \pm 1.3 \%, \mathrm{p}<0.05$ ) (Fig. 1). Because the major component of Clostridium subcluster 4 in the human intestinal microbiota is Clostridium leptum, the relative abundance of Clostridium leptum was investigated by the genus-specific quantitative PCR method. The relative abundance of Clostridium subcluster 4 as shown by the T-RFLP method was significantly correlated with the relative amount of Clostridium leptum measured by the quantitative PCR method (Fig. 2, r $=0.91 ; \mathrm{p}<0.05$ ). Therefore, supplementation of L-carnitine to the patients receiving hemodialysis was found to alter their intestinal microbiota significantly.

\section{DISCUSSION}

Recent findings have shown that the composition of the intestinal microbiota is skewed in patients with chronic kidney disease and end-stage renal disease and that an intervention targeting the microbiota might prevent the progression of renal dysfunction $[6,13]$. As the Lactobacillaceae and Prevotellaceae families were shown to decrease in CKD patients, the supplementation with probiotics including Lactobacillus acidophilus was performed in CKD patients [14]. This treatment reportedly decreased the BUN level in the CKD patients.

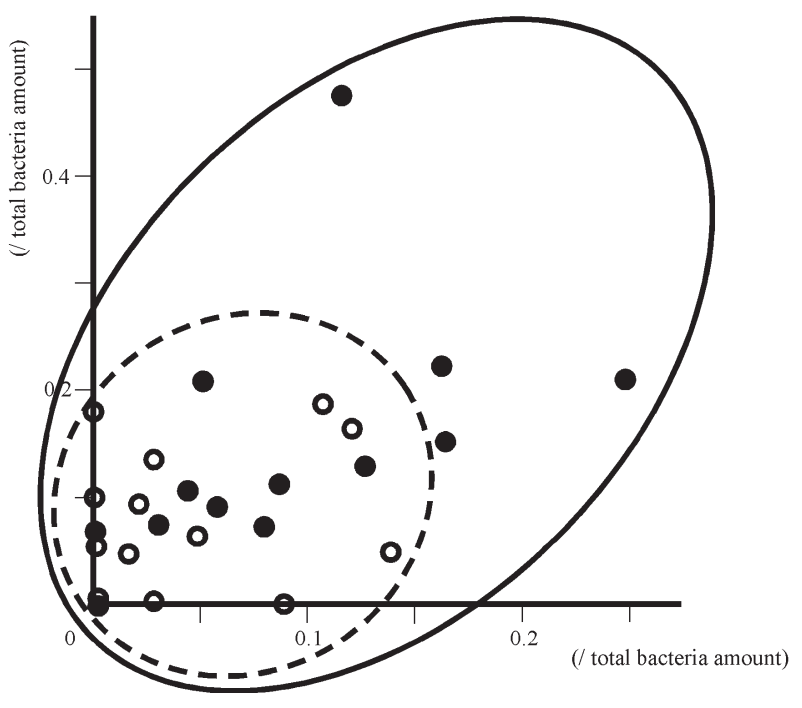

Fig. 2. The relative abundance of intestinal Clostridium subcluster 4 and Clostridium leptum in the hemodialysis patients before and after oral L-carnitine supplementation for 3 months.

The relative abundances of Clostridium subcluster 4 measured by the T-RFLP method are shown on the horizontal axis, and the relative abundances of Clostridium leptum measured by the quantitative PCR method are shown on the vertical axis. Closed circles, before treatment; open circles, after treatment. 
In patients treated with hemodialysis, supplementation with Lactobacillus was found to decrease their serum nitrogen metabolite levels and improve their muscle volume and appetite [15]. These reports suggested that the modulation of dysbiosis found in patients with endstage renal disease would be beneficial for renal function and nitrogen control.

Instead of modulation of the intestinal microbiota by microorganisms, the medication for constipation was shown to also alter the intestinal environment with changes in the microbiota to protect against the progression of CKD [16]. Uremic toxins such as indoles are produced by the intestinal microbiota, and these toxins worsen renal function and the prognosis of end-stage renal disease [17]. We showed that oral supplementation with L-carnitine improved gastrointestinal disorders and nitrogen management in patients receiving hemodialysis. Although the precise mechanism of the improvement induced by L-carnitine is unclear, the improvement in gastrointestinal dysmotility might contribute to the beneficial effect. Further investigation of the underlying mechanism is essential for carnitine therapy.

The limitations of the study are the small number of enrolled patients, the lack of a placebo control, and the insufficient accuracy of the microbiota analysis. Although the number of the patients was limited, the consistency of the intestinal microbiota between before and after treatment indicated that the alternation of specific bacterial species would have important implications. The effect of hemodialysis on the intestinal microbiota should have been excluded by a placebo group. Alternation of the intestinal microbiota, however, was not observed in participants treated with plasmapheresis for hyperlipidemia (data not shown). Thus, the effects of the hemodialysis procedure on the intestinal microbiota might be excluded. In order to investigate the alteration of the intestinal microbiota caused by L-carnitine in detail, a meta-16S rDNA analysis, which was recently developed, should be conducted to determine the specieslevel changes in the intestinal microbiota. Although the analytical method used in this study did not possess the power to detect species-level changes, the observed decrease in Clostridium subcluster 4 was verified by two independent methods. Further studies are needed to clarify which species was changed by the therapy.

\section{CONCLUSION}

In summary, the oral supplementation of L-carnitine to the patients receiving hemodialysis improved not only their muscle discomfort but also their gastrointestinal disorders and microbiota. The effect of supplementation with L-carnitine on the prognosis of hemodialysis patients should be further investigated.

\section{Conflict of interest}

None to declare.

\section{Contribution statement}

J.I., Y.K, R.K., T.Y., S.M, H.I, and M.H. designed the study. J.I., Y.K, R.K., T.Y., S.M, M.W., H.I, and M.H. performed the study. J.I., Y.K, and R.K. analyzed the data. J.I., Y.K, R.K., T.Y., S.M, H.I, and M.H. wrote the manuscript. All of the authors participated in the data interpretation, critically reviewed the manuscript, and approved the final version.

\section{Transparency declarations \\ None to declare.}

\section{ACKNOWLEDGEMENTS}

Part of this study was reported at the Annual Congress of the Japanese Society of Dialysis (Fukuoka, 2013). The authors thank Dr. Edward Barroga, Associate Professor and Senior Editor of the Department of International Medical Communications of Tokyo Medical University, for editing the manuscript and Dr. Katsuyoshi Matsuoka, Associate Professor of the Department of Gastroenterology and Hepatology, Tokyo Medical and Dental University, for reviewing the manuscript.

\section{REFERENCES}

1. Khalatbari-Soltani S, Tabibi H. 2015. Inflammation and L-carnitine therapy in hemodialysis patients: a review. Clin Exp Nephrol 19: 331-335. [Medline] [CrossRef]

2. Dong $\mathrm{R}$, Guo ZY, Ding JR, Zhou YY, Wu H. 2014. Gastrointestinal symptoms: a comparison between patients undergoing peritoneal dialysis and hemodialysis. World J Gastroenterol 20: 11370-11375. [Medline] [CrossRef]

3. Weaver LT, Rosenthal SR, Gladstone W, Winter HS. 1992. Carnitine deficiency: a possible cause of gastrointestinal dysmotility. Acta Paediatr 81: 79-81. [Medline] [CrossRef]

4. Casciani CU, Caruso U, Cravotto E, Corsi M, Maccari F. 1982. Beneficial effects of L-carnirine in postdialysis sundrome. Current Therapeutic Research 32: 116-127.

5. Anders HJ, Andersen K, Stecher B. 2013. The intestinal microbiota, a leaky gut, and abnormal immunity in kidney disease. Kidney Int 83: 1010-1016. [Medline] [CrossRef] 
6. Vaziri ND, Wong J, Pahl M, Piceno YM, Yuan J, DeSantis TZ, Ni Z, Nguyen TH, Andersen GL. 2013. Chronic kidney disease alters intestinal microbial flora. Kidney Int 83: 308-315. [Medline] [CrossRef]

7. Abe M, Kikuchi F, Okada K, Kaizu K, Matsumoto K. 2008. Efficacy of pioglitazone on type 2 diabetic patients with hemodialysis. Diabetes Res Clin Pract 80: 432-438. [Medline] [CrossRef]

8. Nagashima K, Hisada T, Sato M, Mochizuki J. 2003. Application of new primer-enzyme combinations to terminal restriction fragment length polymorphism profiling of bacterial populations in human feces. Appl Environ Microbiol 69: 1251-1262. [Medline] [CrossRef]

9. Larsen N, Vogensen FK, van den Berg FW, Nielsen DS, Andreasen AS, Pedersen BK, Al-Soud WA, Sørensen SJ, Hansen LH, Jakobsen M. 2010. Gut microbiota in human adults with type 2 diabetes differs from non-diabetic adults. PLoS One 5: e9085. [Medline] [CrossRef]

10. Matsuki T, Watanabe K, Fujimoto J, Takada T, Tanaka R. 2004. Use of 16S rRNA gene-targeted group-specific primers for real-time PCR analysis of predominant bacteria in human feces. Appl Environ Microbiol 70: 7220-7228. [Medline] [CrossRef]

11. Nadkarni MA, Martin FE, Jacques NA, Hunter N. 2002. Determination of bacterial load by real-time PCR using a broad-range (universal) probe and primers set. Microbiology 148: 257-266. [Medline] [CrossRef]

12. David LA, Maurice CF, Carmody RN, Gootenberg DB,
Button JE, Wolfe BE, Ling AV, Devlin AS, Varma Y, Fischbach MA, Biddinger SB, Dutton RJ, Turnbaugh PJ. 2014. Diet rapidly and reproducibly alters the human gut microbiome. Nature 505: 559-563. [Medline] [CrossRef]

13. Ramezani A, Raj DS. 2014. The gut microbiome, kidney disease, and targeted interventions. J Am Soc Nephrol 25: 657-670. [Medline] [CrossRef]

14. Ranganathan N, Ranganathan P, Friedman EA, Joseph A, Delano B, Goldfarb DS, Tam P, Rao AV, Anteyi E, Musso CG. 2010. Pilot study of probiotic dietary supplementation for promoting healthy kidney function in patients with chronic kidney disease. Adv Ther 27: 634-647. [Medline] [CrossRef]

15. Simenhoff ML, Dunn SR, Zollner GP, Fitzpatrick ME, Emery SM, Sandine WE, Ayres JW. 1996. Biomodulation of the toxic and nutritional effects of small bowel bacterial overgrowth in end-stage kidney disease using freeze-dried Lactobacillus acidophilus. Miner Electrolyte Metab 22: 92-96. [Medline]

16. Mishima E, Fukuda S, Shima H, Hirayama A, Akiyama Y, Takeuchi Y, Fukuda NN, Suzuki T, Suzuki C, Yuri A, Kikuchi K, Tomioka Y, Ito S, Soga T, Abe T. 2015. Alteration of the intestinal environment by lubiprostone is associated with amelioration of adenine-induced CKD. J Am Soc Nephrol 26: 1787-1794. [Medline] [CrossRef]

17. Lau WL, Kalantar-Zadeh K, Vaziri ND. 2015. The gut as a source of inflammation in chronic kidney disease. Nephron 130: 92-98. [Medline] [CrossRef] 